\title{
In memoriam Professor Dr. med. Dr. rer. nat. Jörg Hermann Peter
}

\author{
In memoriam Professor Dr. med. Dr. rer. nat. Jörg Hermann Peter
}

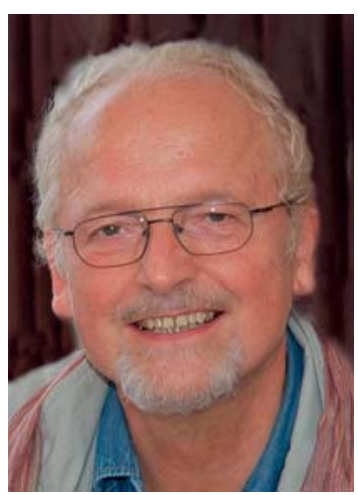

Prof. Dr. med. Dr. rer. nat. Jörg Hermann Peter

\section{Bibliografie}

DOI http://dx.doi.org/

10.1055/s-0029-1243960

Pneumologie 2010; 64:

196-197 @ Georg Thieme

Verlag KG Stuttgart · New York ISSN 0934-8387

Korrespondenzadresse

Prof. Dr. med. Thomas Podszus

Abt. Pneumologie,

Intensivmedizin, Schlafmedizin

Medizinische Klinik

Sana Klinikum Hof

Eppenreuther Str. 9

95032 Hof

Thomas.Podszus@t-online.de
Professor Peter verstarb am 10. Januar 2010 in Marburg, in der Stadt seines jahrzehntelangen Wirkens. Er wurde am 18. Februar 1945 in Treisbach geboren und besuchte die Schule in Marburg. Nach dem Abitur studierte er Humanmedizin und Psychologie; beide Studiengänge schloss er mit einer Promotion ab. Er wurde 1986 zum außerplanmäßigen Professor des Fachbereiches Humanmedizin der Philipps-Universität Marburg ernannt. In seiner Promotionsarbeit im Fach Psychologie beschäftigte er sich mit den Zusammenhängen zwischen Vigilanz, Schläfrigkeit, Leistungsfähigkeit und Veränderungen im autonomen System bei Fahrzeugführern. Nach beruflichen Stationen im Physiologischen Institut sowie im Institut für medizinisch-biologische Statistik der Universität Marburg wechselte er 1979 in die Medizinische Poliklinik, wo er seine Weiterbildung zum Facharzt für Innere Medizin vollzog. 1981 begann Prof. Peter mit dem Aufbau eines Schlaflabors in der damaligen Medizinischen Poliklinik mit dem Ziel, das Krankheitsbild der Schlafapnoe wissenschaftlich zu erforschen. Bereits nach kurzer Zeit war das Aufkommen an behandlungsbedürftigen Patienten derart groß, dass der Schwerpunkt der wissenschaftlichen Tätigkeit auf die Patientenversorgung umdefiniert werden musste. In diesem Rahmen entstand unter seiner Federführung das weltweit erste ambulant einsezbare 4-Kanal-Messgerät, der „Marburger Koffer“, sowie stationär einfach anwendbare 8- und Mehr-Kanalsysteme, mit denen eine zeitgerechte Versorgung der immer größeren Zahl an Patienten möglich wurde. Wissenschaftlich eröffnete sich mit dem Einsatz der Polygraphen die Möglichkeit zu epidemiologischen Untersuchungen, die Prof. Peter bei internistischen Patienten und hier insbesondere bei Patienten mit HerzKreislauf-Erkrankungen erfolgreich durchführte. So entstand als Ergebnis seiner damaligen Arbeit bereits Anfang der 80er-Jahre das Konzept des Risikofaktors der obstruktiven Schlafapnoe für den arteriellen Hypertonus, für die koronare Herzkrankheit sowie für Patienten mit schwerer Herzinsuffizienz. 1985 führte er die nasale Beatmungstherapie für Patienten mit obstruktiver Schlafapnoe in Deutschland ein, zu einem Zeitpunkt, als diese Therapie international noch kontrovers diskutiert wurde. Neben intensiver eigener Forschungstätigkeit gelang es Prof. Peter über 2 Jahrzehnte hinweg eine wissenschaftlich tätige Arbeitsgruppe zu formen und erfolgreich anzuleiten. Hier wurden viele Fragestellungen aus der Atmungs- und Herz-Kreislauf-Physiologie bearbeitet, deren Ergebnisse dank seiner umfassenden Erfahrungen in Physiologie, Methodik und Medizin nationale und internationale Anerkennung erhielten. Durch die von ihm eingebrachte Anwendung moderner statistischer Methoden bei der Zeitreihenanalyse zur Bearbeitung von Biosignalen konnten neue Erkenntnisse in physiologische und pathophysiologische Vorgänge gewonnen werden, die heute bei der Diagnostik und Behandlung verschiedener chronischer Erkrankungen Berücksichtigung finden. Die Fähigkeit, Forschung und Wissenschaft, insbesondere auch unter dem Versorgungsaspekt, erfolgreich durchzuführen sowie wissenschaftliche Aktivitäten in einer Forschergruppe zu koordinieren und immer wieder mit neuen Impulsen zu versehen, haben Prof. Peter weit über die Nation hinaus auch internationale Anerkennung eingebracht. Zwei von ihm organisierte internationale Symposien zu den Themenkomplexen Schlaf, schlafbezogene Atmungsstörungen und Gesundheitsrisiken sowie der Vth World Congress on Sleep Apnea führten in Marburg Ärzte, Forscher und Wissenschaftler aus mehr als 30 Nationen zu intensiven Diskussionen zusammen. Neben ihrer Erforschung hat Prof. Peter die Schlafmedizin in die Arbeit der wissenschaftlichen Fachgesellschaften eingebracht. 1985 initiierte er die Gründung der Arbeitsgruppe nächtliche Atmungs- und Kreislaufregulationsstörungen in der Deutschen Ge- 
sellschaft für Pneumologie, 1991 war er Gründungsmitglied der Deutschen Gesellschaft für Schlafforschung und Schlafmedizin (DGSM), der er in Folgejahren auch als Präsident vorstand. Seine Tätigkeit in wissenschaftlichen Gremien war immer davon gekennzeichnet, dass nur ein interdisziplinärer Denkansatz den Problemen der Schlafmedizin gerecht werden kann. Konsequent lehnte er jeden Wissenschaftspartikularismus ab und suchte immer die Inhalte der Fachgebiete Neurologie, Psychiatrie, Innere Medizin, HNO-Heilkunde, Pädiatrie, Mund-Kiefer-Gesichtschirurgie und Psychologie für eine Optimierung schlafmedizinischer Forschung und Versorgung zusammenzuführen. Für seine wissenschaftlichen Verdienste wurde Prof. Peter zum Ehrenmitglied der Deutschen Gesellschaft für Pneumologie ernannt. In den letzten Jahren war Prof. Peter schwer erkrankt, was zwar seinen beruflichen Einsatz in der Klinik vorzeitig beendete, jedoch seinen Schaffensdrang für die Schlafmedizin nicht aufhalten konnte. So entstanden in dieser für ihn persönlich schweren Zeit die „Enzy- klopädie der Schlafmedizin“ und, redaktionell mitverantwortlich, die „Leitlinie S3 Nicht erholsamer Schlaf/Schlafstörungen“ der DGSM. Diese beiden Werke tragen in unverkennbarer Form seine Handschrift in dem Sinn, dass viele namhafte Autoren aus verschiedenen Gebieten hier eingebunden wurden und eindrucksvoll Prof. Peters interdisziplinären Ansatz belegen. Die Enzyklopädie wie auch die Leitlinie werden in den nächsten Jahren aufgrund ihrer Interdisziplinarität Standardwerke in der deutschen Schlafmedizin sein. Am 10. Januar 2010 starb Prof. Peter plötzlich. Die Schlafmedizin in Deutschland und die in ihr tätigen Fachgebiete verlieren einen Vorreiter. Sein Humor, seine konstruktive und zusammenführende Kritik, seine Anregungen werden fehlen. Seine Ideen, für die er erfolgreich nahezu 30 Jahre gekämpft hat, werden in der Schlafmedizin weiterleben. Die Deutsche Gesellschaft für Pneumologie wird ihr Ehrenmitglied Prof. Dr. Dr. Jörg Hermann Peter in ehrendem Angedenken behalten. 